\title{
Computer Aided Diagnosis For the Detection of Lung Nodules
}

\author{
${ }^{*}$ B.S Yogananda ${ }^{1}$ Dr.Mohana H.S ${ }^{2}$ G. Shivakumar ${ }^{3}$ \\ ${ }^{1}$ Lecturer,Dept. of EEE,Sri Siddhartha Institute of Technology,Tumkur \\ ${ }^{2}$ Professor, Dept. of IT,Malnad College of Engineering, Hassan, Karnataka \\ ${ }^{3}$ Asst. Professor,Dept. of IT,Malnad College of Engineering, Hassan, Karnataka
}

\begin{abstract}
Lungs segmentation is a vital step for computer aided diagnosis. Many lungs diseases requiring radiological support for diagnostic purpose, including tuberculosis, emphysema and lungs cancer could be affectively diagnosed using computer-aid. Pulmonary nodules usually act as the early stage characterization of lung cancer. It's a crucial way to control the disease and reduce the mortality by further examination on the diagnosis of pulmonary nodules. But the large number of the image data and lesion analysis make difficulties to radiology expert for the correctness and effectiveness. So the computer aided detection for the pulmonary nodules turns out to be the hot subject of medical image processing.

In this paper, we follow the classic step in detecting the nodules and we propose an effective algorithm based on the morphology and spatial relationship to search the lesion.This proposal provides an extremely effective way to locate the nodules.
\end{abstract}

Keywords: - pulmonary nodule; computer-aided detection; CT image; morphology; special.

\section{Introduction}

The study indicates that lung cancer has possessed the maximum rate of cancer death across the world. And the results of the studies also show that if the patients could be treated at the early stage of lung cancer, they could have more chance to survive or be endurance. Pulmonary nodules are the characterization of the early stage of the lung cancer.

Computerized Tomography which is widely used in the lung disease check could be the most useful tool to detect the nodules. Several studies demonstrated that $90 \%$ of peripheral lung cancers were visible in CT images earlier than the date of the cancer discovery by the radiologist. When scanning the CT images experts should distinguish the real nodules from the interference region by their knowledge. Due to the fact that the nodules are not simply laying on the images, there are disruptor structures like blood vessels, alveoli and so on as well. Thus experts propose the computer-aided detection CAD method which could assist radiologists to detect pulmonary nodules. The computer-aided detection could improve the accuracy of identifying the nodules and reduce the image scanning time. It is generally considered as a useful tool to provide an advisory opinion to assist the experts' diagnosis.

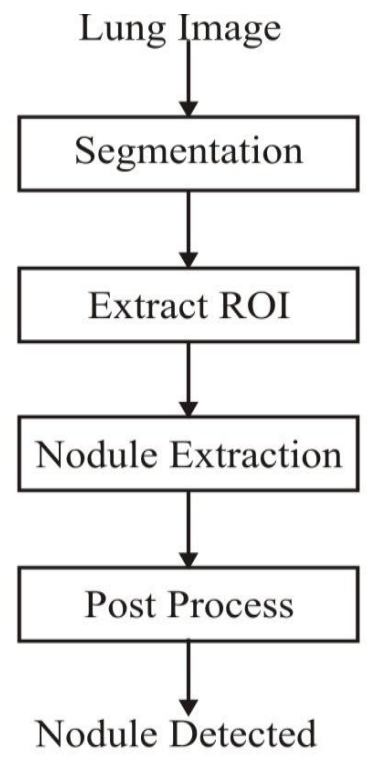

Fig. 1. Block Diagrm 


\section{Review of previous papers}

Some of the technical papers under lung cancer are studied and reviewed to understand the available theories and techniques and to implement alternative methods of solving the above problem which is our objective. Some of the papers we have referred are presented here. We also provide the limitations of those methods and how our approach seems to be advantageous over them.

In [1], Detection of lung cancer form the analysis of computed tomography (CT) images of chest. To produce a successful Computer Aided Diagnosis system, several problems has to be resolved. Segmentation is the first problem to be considered which helps in generation of candidate region for detecting cancer nodules. The second problem is identification of affected nodules from all the candidate nodules. Initially, the basic image processing techniques such as Bit-Plane Slicing, Erosion, Median Filter, Dilation, Outlining, Lung Border Extraction and Flood-Fill algorithms are applied to the CT scan image in order to detect the lung region. Then the segmentation algorithm is applied in order to detect the cancer nodules from the extracted lung image.

In [2], The authors presents an efficient algorithm for segmenting different types of pulmonary nodules including high and low contrast nodules, nodules with vasculature attachment, and nodules in the close vicinity of the lung wall or diaphragm. The algorithm performs an adaptive sphericity oriented contrast region growing on the fuzzy connectivity map of the object of interest.

In [3], The authors presents an automated lung segmentation method has been applied (1) as preprocessing for automated lung nodule detection and (2) as the foundation for computer-assisted measurements of pleural mesothelioma tumor thickness. The core method uses gray-level thresholding to segment the lungs within each computed tomography section.

In [4], Authors developed a computer-aided diagnosis (CAD) system for lung nodule detection on thoracic helical computed tomography (CT) images. In the first stage of this CAD system, lung regions are identified by a k-means clustering technique. Each lung slice is classified as belonging to the upper, middle, or the lower part of the lung volume. Within each lung region, structures are segmented again using weighted kmeans clustering

From the above methods, Pulmonary nodules needs to be separated from larger lung tumors, smaller infiltrates or masses with other accompanying characteristics, i.e. Nodule boundary. Processing time is more as ROI is not accurate. Different CAD applications impose different requirements on the automated lung segmentation process. The specific approach to lung segmentation must be adapted to the particular CAD task. To overcome the above problems, the following method is proposed.

\section{Methodology}

Computer-Aided Detection is classical and easy going but not too following the beaten path. It is suited to solute the problem. The morphological operations have been applied here. The tissue or blood vessels around the lung would be involved, but it matters nothing as they would be dealt with by following treatment.

From the morphological aspect, the pulmonary nodules have a variety of uncertain forms that is due to the different growing environment of the nodules. But it often takes the circle and ellipse as the main state. And the blood vessels usually turn out to be striped or bifurcated. Thus we could separate the most blood vessels from the nodules through detecting the characteristic.

The lung CT images complex information such as the patients' basic information, the materials around the body and truck tomography which is the region our research focused on. To establish a good environment for detecting the nodules we must get rid of the unrelated region and make the lung region complete as well.

The lung anatomical structure and spatial characteristics performs well in distinguishing nodules and provides relatively fast speed.

\section{Result Discussion}

The graphical user interface of our project is as shown in fig.2 and fig.3. In our project, we have used .Net framework to develop front end of our project.

In our project to detect pulmonary nodules, initially we have taken the CT scan of a patient, which is available from digital libraries at universities.

The CT scan is converted into Gray scale image for further processing as shown in fig 2 . Then the lung ROI and lung outline region is detected using morphological process and finally using spatial characteristics we differentiate the nodules from blood vessels, which may have the similar values as nodules. And finally we get the pulmonary nodules, as shown in fig 3 . 


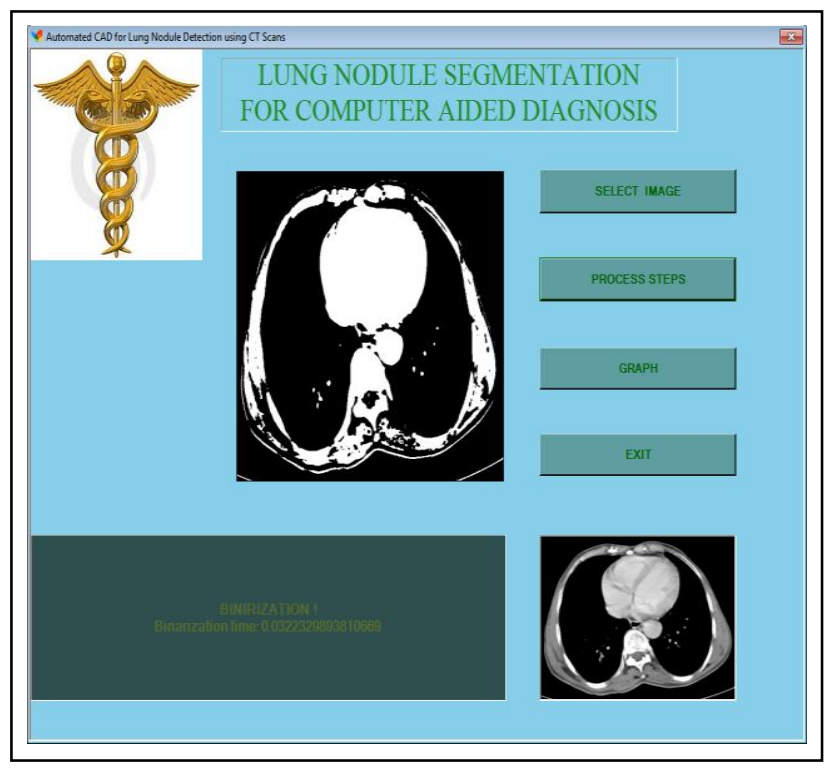

Fig 2. Gray scale conversion.

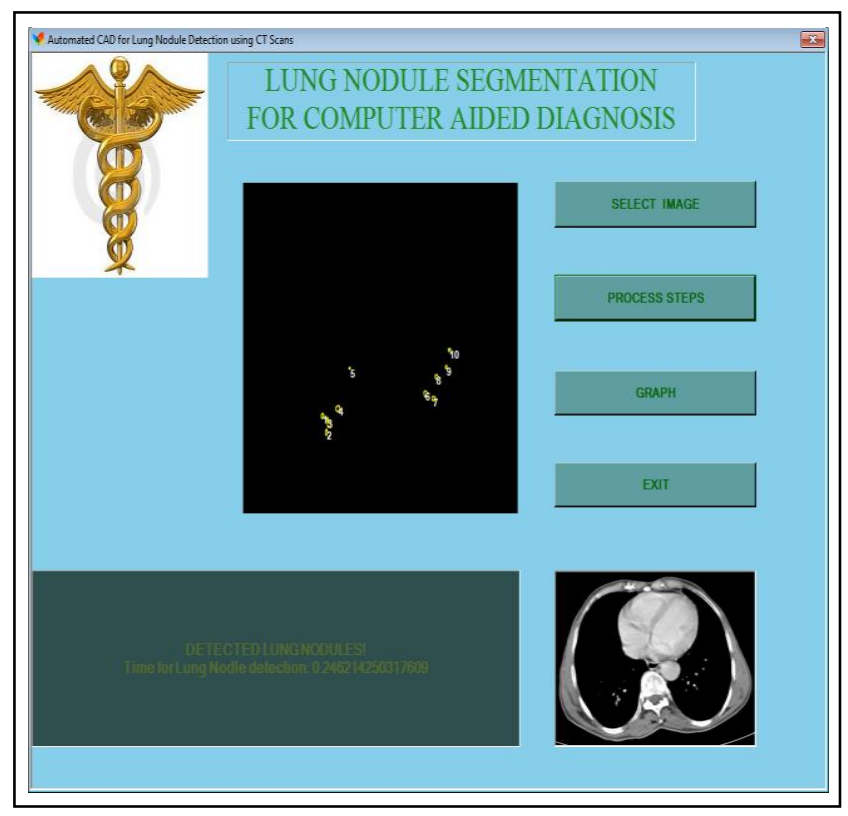

Fig 3. Nodules detected.

\section{Conclusion}

In our project pulmonary nodules have been successfully detected using morphological and spatial methods. And from the survey it is found that the obtained result have shown less false detection of nodules.

\section{References}

[1] Cancer facts and figures 2005, American Cancer Society, http://www.cancer.org.

[2] G. L. Zorn and J. C. Nesbitt, "Surgical management of early stage lung cancer," Seminars in Surgical Oncology 18, 124-136, 2000.

[3] J.H.M. Austin, B.M. Romeny, and L.S. Goldsmith, "Missedbronchogenic carcinoma: radiographic findings in 27 patients with apotentially respectable lesion evident in retrospect”, Radiology 182, pp.115-122, 1992.

[4] Erberich SG, Song KS, Arakawa H, Huang HK, Webb R, Hoo KS, Loo BW. Knowledge-Based Lung Nodule Detection from Helical CT. Bildverarbeitung fr die Medizin, 1999, pp 337-341.

[5] Kanazawa K, Kawata Y, Niki N, Satoh H, Ohmatsu H, Kakinuma R, Kaneko M, Moriyama 